\title{
Characterization of Mce4A protein of Mycobacterium tuberculosis: role in invasion and survival
}

\author{
Neeraj Kumar Saini ${ }^{1}$, Monika Sharma ${ }^{1}$, Amita Chandolia ${ }^{1}$, Rashmi Pasricha ${ }^{1}$, \\ Vani Brahmachari ${ }^{2}$ and Mridula Bose*1
}

Address: ${ }^{1}$ Department of Microbiology, Vallabhbhai Patel Chest Institute, University of Delhi, Delhi, India and ${ }^{2}$ Ambedkar Center for Biomedical Research, University of Delhi, Delhi, India

Email: Neeraj Kumar Saini - neeraj_k_saini@yahoo.co.in; Monika Sharma - monika_sharma18@rediffmail.com;

Amita Chandolia - amita.micro@gmail.com; Rashmi Pasricha - rashmipasricha@gmail.com; Vani Brahmachari - vani.brahmachari@gmail.com; Mridula Bose* - mridulabose@hotmail.com

* Corresponding author

Published: 19 November 2008

BMC Microbiology 2008, 8:200 doi:10.1/86/|47|-2180-8-200
Received: 12 June 2008

Accepted: 19 November 2008

This article is available from: http://www.biomedcentral.com//47|-2/80/8/200

(c) 2008 Saini et al; licensee BioMed Central Ltd.

This is an Open Access article distributed under the terms of the Creative Commons Attribution License (http://creativecommons.org/licenses/by/2.0), which permits unrestricted use, distribution, and reproduction in any medium, provided the original work is properly cited.

\begin{abstract}
Background: The mce4 operon is one of the four homologues of mammalian cell entry (mce) operons of Mycobacterium tuberculosis. The mce4A (Rv3499c) gene within this operon is homologous to mce IA (Rv0/69), that has a role in host cell invasion by M. tuberculosis. Our earlier reports show that mce 4 operon is expressed during the stationary phase of growth of the bacillus in culture and during the course of infection in mammalian hosts. M. tuberculosis carrying mutation in mce4 operon shows growth defect and reduced survival in infected mice. However, the intracellular localization of Mce4A protein and its direct role in cell entry or survival of the bacillus has not been demonstrated so far.

Results: By transmission electron microscopy we have demonstrated that recombinant Mce4A protein facilitates the invasion of non-pathogenic strain of $E$. coli into non-phagocytic HeLa cells. We observe that mce $4 A$ gene has a role comparable to mce $I A$ in the survival of recombinant $E$. coli in human macrophages. Using antibodies raised against Mce4A protein, we show that the protein is localized in the cell wall fraction of $M$. tuberculosis H37Rv stationary phase culture only.

Conclusion: Mce4A protein is expressed during the stationary phase of broth culture and localizes in the cell wall fraction of $M$. tuberculosis. Mce4A protein expressed in non-pathogenic $E$. coli enables it to enter and survive within HeLa cells and the macrophages. As Mce4A protein is expressed during later phase of mycobacterial growth, our results raise the possibility of it playing a role in maintenance of persistent tubercular infection.
\end{abstract}

\section{Background}

The World Health Organization has estimated that nearly one-third of the world's population is now latently infected with Mycobacterium tuberculosis and 8-10 million people develop active disease resulting in 2 million deaths each year $[1,2]$. The prevalence of this infection is largely due to the extended dormancy of $M$. tuberculosis in the host and its ability to cause disease even in the face of a highly orchestrated host immune response [3].

M. tuberculosis is believed to primarily invade and replicate within alveolar macrophages [4]. It has the ability to enter 
A549 cells of alveolar epithelial origin in culture [5] and also invade other non-phagocytic cells $[6,7]$.

Role of Mce1A protein in the entry and survival of the pathogen is demonstrated using recombinant $E$. coli carrying cloned copy of the gene as well as latex microspheres coated with recombinant Mce1A protein, both of which were able to enter HeLa cells $[8,9]$. Additionally, it has been demonstrated that the recombinant $E$. coli expressing Mce1A can survive longer in these cells [8]. The continued analysis of the four mce operons of $M$. tuberculosis H37Rv genome has shown significant conservation of the protein sequences in the four operons [10]. The redundancy of these operons is explained by functional significance of the different mce operons of $M$. tuberculosis H37 Rv by various studies including ours [11-15]. Earlier we reported the expression of mce4 and absence of mce1 transcripts in the specific tissues of both infected rabbits and guinea pigs during advanced disease conditions, which suggested that mce4 operon, may have a role in the long term survival of $M$. tuberculosis in host's tissue [11]. The survival mechanism of dormant tubercle bacilli is still under intense research [16-18]. It is suggested that mycobacterial persistence may rise as a response to environmental stress, such as reduced oxygen concentration within a host tissue [19]. It is demonstrated that deletion mutants of mce3 and mce4 operons of M. tuberculosis $\mathrm{H} 37 \mathrm{Rv}$ are attenuated in mice [13]. By deleting eight genes and the first $250 \mathrm{bp}$ of the ninth gene from mce 4 operon it was shown that survival of $M$. tuberculosis was significantly reduced in mice [13]. However, direct involvement of individual proteins from mce4 operon in the survival or uptake of $M$. tuberculosis into the mammalian cells was not demonstrated.

In the present study we validate the role of Mce 4A protein of mce4 operon not only in cell invasion but also in survival of the pathogen in human macrophages using recombinant $E$. coli expressing cloned mce4A gene. The cellular localization and in vitro expression of the Mce4A protein in M. tuberculosis was also analyzed.

\section{Methods}

\section{Bacterial strains, cells and culture conditions}

M. tuberculosis H37Rv was grown as shake-cultures in Middlebrook 7H9 broth (Difco) supplemented with OADC (oleic acid, albumin [bovine, fraction V], dextrose, catalase [Difco]) and $2 \%$ glycerol at $37^{\circ} \mathrm{C}$.

E. coli DH5 $\alpha$ and BL-21 (DE3) cells were grown in LuriaBertani (LB) broth or on LB agar in presence of kanamycin $(25 \mu \mathrm{g} / \mathrm{ml})$ where ever appropriate. The human epithelial cell line HeLa cells were grown in complete Dulbecco's modified Eagle's medium (DMEM, Gibco) supplemented with $10 \mathrm{mM}$ sodium pyruvate (Sigma), 10\% FBS (fetal bovine serum, Gibco), HEPES (Sigma) and $10 \mu \mathrm{g}$ of penicillin-streptomycin/ml (Sigma). THP-1 monocyte cell line was cultured in RPMI 1640 (Sigma) supplemented with $10 \%$ FCS (fetal calf serum, Gibco) and $2 \mathrm{mM} \mathrm{L-}$ glutamine (Sigma). Both the cell lines were grown at $37^{\circ} \mathrm{C}$ in presence of $5 \% \mathrm{CO}_{2}$. PMA (phorbol myristate acetate, Sigma) was used for the adhesion of THP-1 cells.

Cloning, expression and purification of Mce IA and Mce4A Genomic DNA from $M$. tuberculosis H37Rv was extracted according to the CTAB (cetyltrimethylammonium bromide) method [20]. Full length mce1A (Rv0169, 1365 bp) and mce4A (Rv3499c, 1203 bp) genes were amplified from M. tuberculosis genomic DNA [21] by polymerase chain reaction (PCR) using the primers mce1F and mce1 $R$ for $m c e 1 \mathrm{~A}$ gene, mce $4 \mathrm{~F}$ and mce4R for $m c e 4 \mathrm{~A}$ gene as listed in Table 1. Each forward primer contained SacI restriction enzyme site whereas the reverse primers contained a HindIII restriction site.

The amplicons were cloned in pET28a (Novagen) to generate pET28a/mce1A and pET28a/mce4A with hexa histidine tags. Both DH5 $\alpha$ and BL-21 (DE3) strains of E. coli were transformed with the recombinant plasmids for expression studies. The presence of the inserts was confirmed by restriction enzyme digestion and sequencing.

Overnight grown culture of E. coli BL-21 (DE3) containing the recombinant expression plasmids was diluted $1: 10$ in fresh LB broth containing kanamycin and grown with vigorous shaking at $37^{\circ} \mathrm{C}$ to an optical density (OD) of 0.6 at $600 \mathrm{~nm}$. For transcription induction, IPTG (isopropyl

Table I: Primers used for PCR amplification of mce IA and mce4A gene.

\begin{tabular}{llc}
\hline Primer* & Sequence $\left(5\right.$ ' to $\left.3^{\prime}\right)$ & Gene \\
\hline mcelF & ATCTCACGGTGTGAGCTCATGACGACGCCGG & mce IA \\
mcelR & CGACGG TTCCAGAAGCTTTCATGGGTTGAT & mce4A \\
mce4F & CTTCAGAAAGAGCTCATGTCCGGCGGCGGA & \\
mce4R & CGCCCGAGCAAGCTTTCAGAAGTCGTCCCG & \\
\hline
\end{tabular}

* Restriction sites in primers are indicated in bold letters. 
thio-b-D-galactoside, Sigma) to a final concentration of $0.5 \mathrm{mM}$ was added to the culture and incubation was continued for $2 \mathrm{~h}$. The N-terminal His-tagged recombinant proteins were purified under denaturing condition on nitrilotriacetic acid (Ni-NTA) agarose columns (Qiagen) according to the manufacturer's instructions.

\section{Production of polyclonal antibodies against Mce4A protein}

The antibodies against the Mce4A protein were raised in 5-month-old female NZW rabbit. For each immunization, $300 \mu \mathrm{g}$ of protein in $0.5 \mathrm{ml}$ PBS was mixed with $0.6 \mathrm{ml}$ of incomplete Freund's adjuvant (Sigma) and administered subcutaneously at five sites in rabbit, followed by four booster injections of $300 \mu$ g each 2, 3, 4 and 6 weeks after the first injection. Serum was prepared from blood collected 2 weeks after the last booster dose. The animal handling was according to the guidelines of the Institutional Ethical Committee and Indian Council of Medical Research, India.

\section{Preparation of subcellular fractions}

M. tuberculosis H37Rv was grown in one liter of $7 \mathrm{H} 9$ Middlebrook medium to mid-log phase (day 6) at $37^{\circ} \mathrm{C}$ on shaking $(200 \mathrm{rpm})$ up to an OD of 0.6 at $600 \mathrm{~nm}$. Cells were harvested by centrifugation at $10,000 \mathrm{~g}$ for $30 \mathrm{~min}$ and the culture supernatant was concentrated to obtain the culture filtrate protein (CFP). Pelleted cells were washed, resuspended in PBS and subcellular fractions were prepared as previously described [22,23]. Similarly, cellular fractions were also prepared from the stationary phase culture (day 10, OD of 1.0 at $600 \mathrm{~nm}$ ) of M. tuberculosis H37Rv.

\section{Detection of protein expression}

The protocol followed for Western blotting of purified Mce $1 \mathrm{~A}$ and Mce4A proteins was as described previously [24]. Anti-His monoclonal antibody (1:2000 dilution) and peroxidase-conjugated goat anti-mouse IgG (1:2000) were used as primary and secondary antibody respectively. Western blot was carried out to detect purified Mce4A protein and also the expression of Mce4A protein in the whole cell lysate of cells harvested on day 2 (OD0.2 at $600 \mathrm{~nm}$ ), day 4 (OD-0.4), day 7 (OD-0.7), and day 10 (stationary phase, OD-1.0) post inoculation. Polyclonal anti-Mce4A antibody (1:2000) raised in rabbits, was used as the primary antibody. For the intracellular localization of Mce4A protein in M. tuberculosis, Western blotting was performed with cytoplasmic (C), cell membrane $(\mathrm{CM})$, cell wall $(\mathrm{CW})$ fractions, culture filtrate protein (CFP) and whole cell lysate (CL) prepared from both log and stationary phase culture as described above.

\section{Invasion assay and Electron microscopy}

Invasion of HeLa cells seeded at $2 \times 10^{6}$ cells per well into 6 -well plates by BL-21 cells transformed with pET28a/ $m c e 1 A$, pET28a/mce4A and empty vector pET28a was assayed. Bacterial cultures grown overnight were diluted $(1: 10)$ in fresh medium containing kanamycin and incubated further with vigorous shaking to reach an OD of 0.6 at $600 \mathrm{~nm}$ and induced with $0.5 \mathrm{mM}$ IPTG for $2 \mathrm{~h}$ at $37^{\circ} \mathrm{C}$. Following the induction, the cells were washed once with PBS and resuspended in $5 \mathrm{ml}$ of PBS. Prior to the infection, monolayer of HeLa cells were charged with fresh medium (DMEM, supplemented with 10\% FCS) and incubated at $37^{\circ} \mathrm{C}$ for $30 \mathrm{~min}$. Recombinant E. coli cells were added to the monolayer at a multiplicity of infection (MOI) of $10: 1$ and incubated at $37^{\circ} \mathrm{C}$ for $4 \mathrm{~h}$, washed three times with PBS and processed for electron microscopy as described previously [9] and examined under CM10 electron microscope. Since the E. coli strain used is kanamycin resistant, the cells were not treated with kanamycin or gentamycin but were extensively washed postinfection to remove the extra-cellular bacteria that failed to enter the mammalian cells.

\section{Survival assay}

THP-1 cells were seeded at $2 \times 10^{6}$ cells per well into 12 well plates. PMA (20 ng/ml) was added for the cell adhesion. Recombinant bacterial cultures induced with IPTG were incubated with THP-1 cells at a MOI of 10:1. Prior to the infection, THP-1 cells were charged with fresh medium (RPMI supplemented with $10 \%$ FCS and $2 \mathrm{mM}$ L-glutamine) and incubated at $37^{\circ} \mathrm{C}$ for $30 \mathrm{~min}$. Infection was continued for $1 \mathrm{~h}$ at $37^{\circ} \mathrm{C}$.

Following $1 \mathrm{~h}$ incubation, the cells in all the wells were washed three times with FCS free RPMI. One set of cells were lysed immediately after $1 \mathrm{~h}$ of incubation. The CFU at this time point was taken as $\mathrm{t}_{0}$ (CFU at time 0 ). The other sets were lysed after $3 \mathrm{~h}\left(\mathrm{t}_{3}\right)$ and $24 \mathrm{~h}\left(\mathrm{t}_{24}\right)$ of incubation. Briefly, the cells were incubated for $10 \mathrm{~min}$ in 500 $\mu \mathrm{l}$ of lysis buffer (0.1\% Triton X-100 in PBS, pH 7.4). The lysate was plated on $\mathrm{LB}$ agar plates and incubated at $37^{\circ} \mathrm{C}$ overnight and recombinant $E$. coli colonies were counted. The numbers of E. coli able to survive at $3 \mathrm{~h}$ and $24 \mathrm{~h}$ post infection were normalized against CFU at $t_{0}$ and expressed as percentage survival. Experiment was done in triplicate.

\section{Statistical analysis}

The CFU of recombinant E. coli and the percentage survival of recombinant $E$. coli cells are represented as mean \pm standard deviation. The difference in percentage survival of recombinant E. coli cells (pET28a/mce1A and pET28a/ $m c e 4 A$ ) as compared to E. coli (pET28a) was calculated using student's t test using graph pad prism ${ }^{\mathrm{Tm}}$ software. 


\section{Results \\ Cloning, expression and purification of Mce IA and Mce4A proteins}

The expression of the fusion proteins was studied in E. coli BL-21 (DE3) strain. The His-tagged Mce1A and Mce4A were expressed at high level on induction with IPTG and the purified proteins were detected using anti-His-tag monoclonal antibody (Fig. 1B). The fusion proteins were of the expected size of $45 \mathrm{kDa}$ and $43 \mathrm{kDa}$ respectively (Fig. 1A). Further we confirmed the identity of purified Mce4A protein with rabbit anti-Mce4A polyclonal antibodies raised in our laboratory (Fig. 2A). Mce4A was purified as a single band, while Mce1A was detected as a doublet. Since anti-His-tag antibodies detected the lower molecular weight band, it is probably due to partial degradation. Similar profile for Mce1A protein was reported earlier also [25].

\section{Growth-phase dependent expression and subcellular localization of Mce4A protein of M. tuberculosis}

Immunochemical detection of Mce $4 \mathrm{~A}$ protein in whole cell lysate from different phase of $M$. tuberculosis cultures shows that it is expressed only in stationary phase culture (data not shown) and the subcellular fractions from stationary phase culture show that Mce4A is localized in cell wall fraction only (Fig. 2B). In a similar experiment subcellular fraction from mid-log phase culture of M. tuberculosis H37Rv, antibodies against Mce4A did not detect any protein either in whole cell lysate or any subcellular fraction (data not shown), which indicates absence of expression of Mce 4A during early phase of growth.

\section{Invasion of HeLa cells by recombinant E. coli expressing Mce4A protein}

The invasion of HeLa cells by recombinant E. coli was monitored by transmission electron microscopy. The results show that uninduced E. coli (pET28a/mce4A and
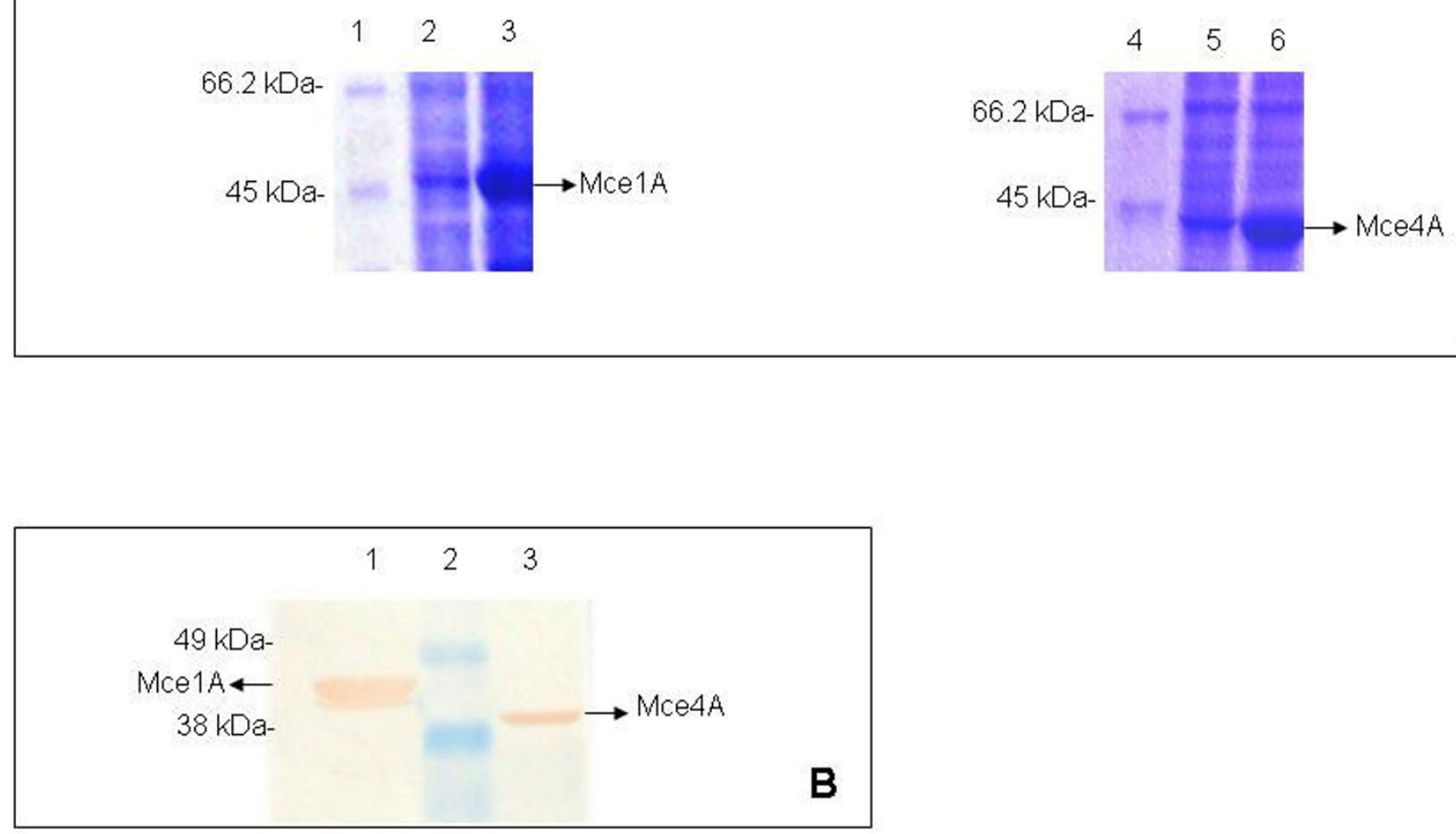

\section{Figure I}

SDS-polyacrylamide gel electrophoresis and Immunoblot analysis. (A) SDS-polyacrylamide gel electrophoresis of whole-cell lysates of uninduced and induced recombinant $E$. coli cells. Lane I, 4- Molecular mass marker (I I6 kDa, 66.2, 45, 35, 25 , I8.4, I4.4, Fermentas), the molecular mass standards are indicated in kDa on left side. Lane 2, 3- Lysate of uninduced and induced $E$. coli (pET28a/mcelA) cells respectively. Lane 5, 6- Lysate of uninduced and induced $E$. coli (pET28a/mce4A) cells respectively. Mce IA and Mce4A are indicated by arrows. (B) Immunoblot of purified Mce IA and Mce4A proteins. Anti-His-tag antibody was used to demonstrate the fusion proteins. Lanel-Purified McelA protein $(2 \mu g)$. Lane 2- Pre-stained molecular mass marker (I88 kDa, 62, 49, 38, 28, I8, I4, 6, 3, Invitrogen), the molecular mass standards in kDa are indicated on left side. Lane 3-Purified Mce4A protein $(2 \mu \mathrm{g})$. Mce IA and Mce4A are indicated by arrows. 

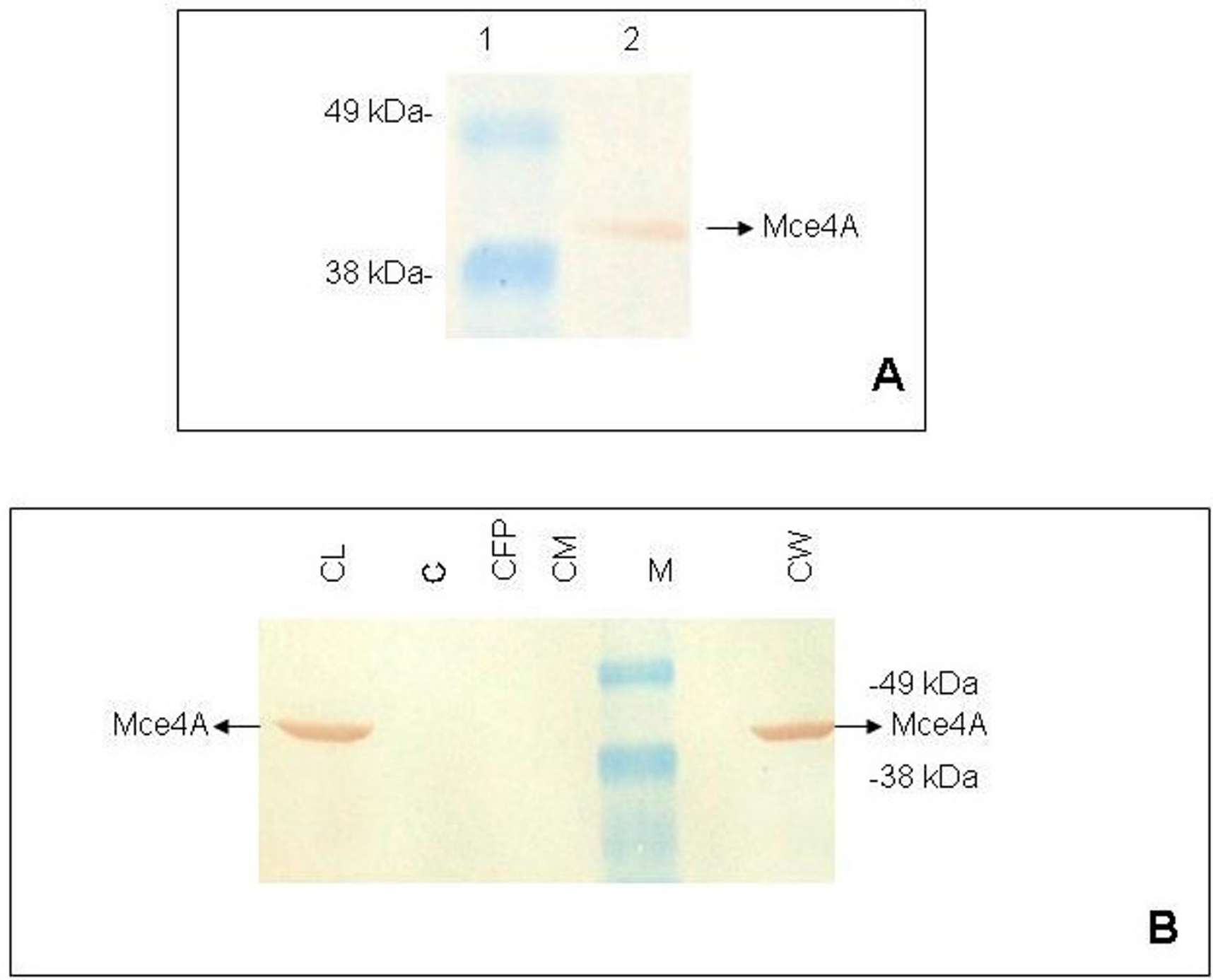

\section{Figure 2}

Immunoblot analysis of Mce4A protein. (A) Purified Mce4A protein separated by SDS-PAGE and electrotransferred to nitrocellulose membrane. Rabbit polyclonal antibody to Mce4A protein used at a dilution of $1: 2000$, detected signal of purified Mce4A protein. Lane I-Pre-stained molecular mass marker (I88 kDa, 62, 49, 38, 28, 18, 14, 6, 3, Invitrogen), the molecular mass standards are indicated in kDa on left side. Lane 2: Purified Mce4A protein $(\mathrm{I} \mu \mathrm{g})$. Mce4A protein is indicated by arrow. (B) Subcellular fractions of $M$. tuberculosis H37Rv, stationary phase culture were fractionated on SDS-PAGE and Western blotting was performed using polyclonal anti-Mce4A antibodies for localization of Mce4A in various fractions. Concentration of protein loaded per well is indicated in parenthesis. Lane CL-Whole cell lysate $(50 \mu \mathrm{g})$, Lane C-Cytoplasm (40 $\mu \mathrm{g})$, Lane CFPCulture filtrate proteins $(30 \mu \mathrm{g})$, Lane CM-Cytoplasmic membrane ( $30 \mu \mathrm{g})$, Lane M-Pre-stained molecular mass marker (I 88 $\mathrm{kDa}, 62,49,38,28,18,14,6,3$, Invitrogen), the molecular mass standards are indicated in $\mathrm{kDa}$ on right side, Lane CW-Cell wall fraction $(30 \mu \mathrm{g})$. Mce4A protein is detected only in the whole cell lysate and cell wall fraction from stationary phase culture.

pET28a/mce1A) (Fig. 3A: a and 3B: f respectively) were not able to enter the HeLa cells while IPTG induced recombinant $E$. coli expressing Mce4A protein (Fig. 3A: c-e) and Mce1A protein (Fig. 3B: h-i) were able to invade the HeLa cells. The invaded HeLa cells showed membrane-ruffling, presence of multiple invaginations at the cell membrane and multiple vacuolations in the cells (Fig. 3A: b-e and $3 \mathrm{~B}: \mathrm{g}, \mathrm{i})$. Internalized recombinant $E$. coli were observed within the cytoplasmic vacuoles (Fig. 3A: c-e and 3B: hi). The E. coli (pET28a) used as a negative control failed to enter the HeLa cells (Fig. 3C: j). 

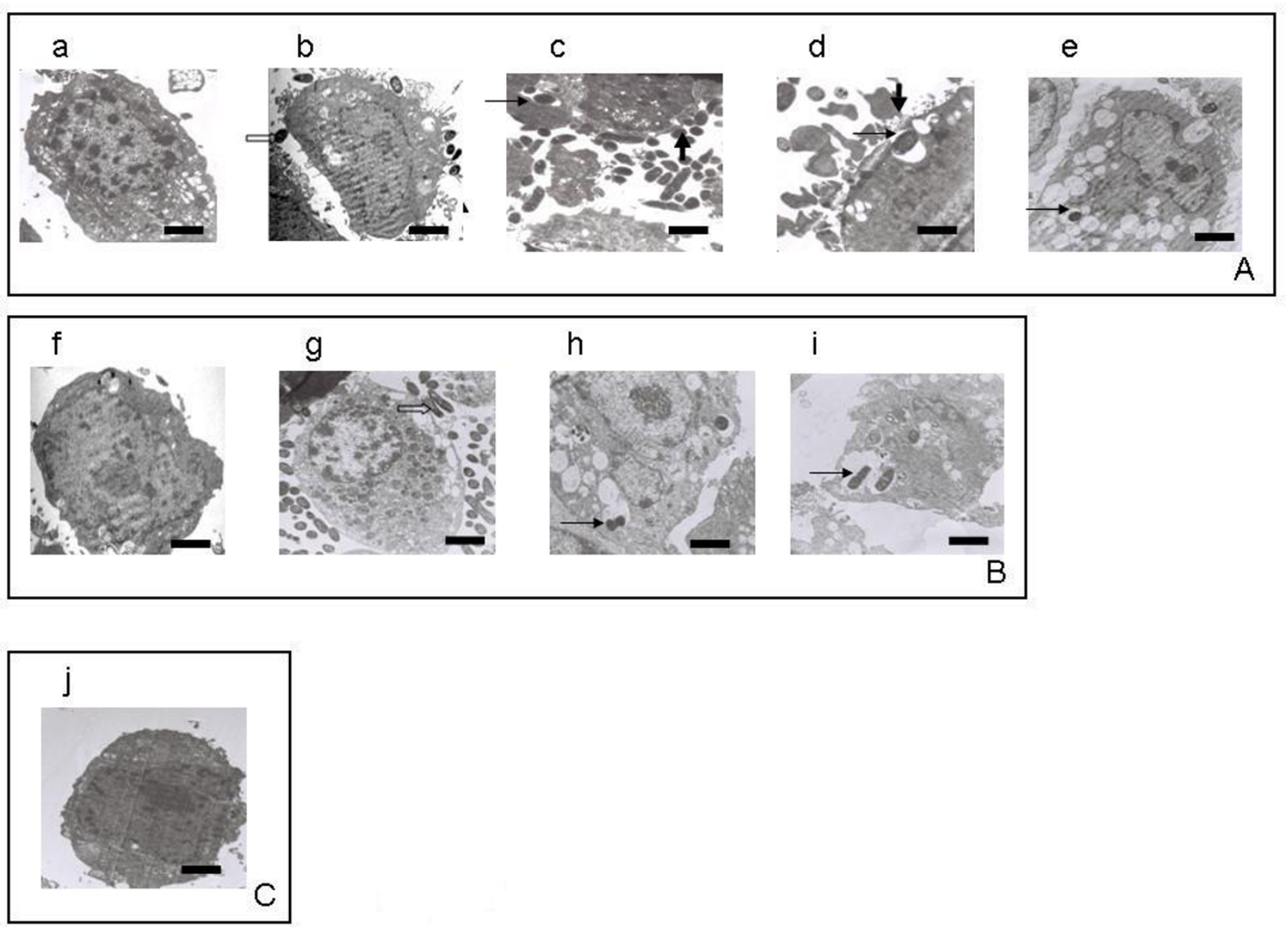

\section{Figure 3}

Invasion of HeLa cells by recombinant $E$. coli. The invasion of HeLa cells by $E$. coli expressing Mce4A and Mce IA proteins was monitored by transmission electron microscopy. The stages of invasion by recombinant $E$. coli (pET28a/mce4A) (fig. 3A), $E$. coli (pET28a/mce IA) (fig. 3B) and E. coli (pET28a) (fig. 3C: j) are shown. A: a and B: f infection with uninduced recombinant $E$. coli. A: b, c, d, e and B: $g$, h, i infection with induced recombinant E. coli expressing Mce4A and Mce IA respectively. Unfilled arrow (bacteria attached to cell membrane), thick arrow (membrane ruffling), thin arrow (internalized bacteria). Bars $=\mathrm{I} \mu \mathrm{m}$ in all the cases, except in $A: c, d$ and $B: g, h$, where it is $0.1 \mu \mathrm{m}$.

\section{Role of Mce4A protein in survival of E. coli in the THP-I macrophages}

To determine the role of Mce4A protein in survival of $M$. tuberculosis within macrophages, the THP-1 cells were infected with recombinant $E$. coli expressing Mce4A or Mce1A at MOI of 10:1 and percentage survival was determined at $3 \mathrm{~h}$ and $24 \mathrm{~h}$ post-infection. The percentage survival at $3 \mathrm{~h}$ post-infection of E. coli (pET28a/mce1A) and E. coli (pET28a/mce4A) was significantly higher than that of $E$. coli (pET28a) at the same time points post-infection ( $\mathrm{p}<0.005)$ (Table 2$)$. At $24 \mathrm{~h}$ post-infection, negligible number of colonies of E. coli (pET28a) was obtained from lysed cells as compared to that from E. coli (pET28a/ mce $1 A, \mathrm{p}<0.0005)$ and E. coli (pET28a/mce $4 A \mathrm{p}<0.005)$
(Table 2). At $3 \mathrm{~h}$ and $24 \mathrm{~h}$ percentage survival of E. coli (pET28a/mce4A) was comparable to that of E. coli (pET28a/mce1A) (p > 0.05 at $3 \mathrm{~h}$ and $24 \mathrm{~h})$.

\section{Discussion}

The initial step in the pathogenesis by intracellular pathogens is the invasion of host cells. In case of M. tuberculosis, Mce1A protein of mce 1 operon was the first protein of the pathogen to be implicated in invasion and survival inside macrophages [8]. Although Mce2A protein of mce2 operon exhibits $67 \%$ amino acid identity with Mce $1 \mathrm{~A}$, latex beads coated with Mce2A are not internalized by HeLa cells [9]. In the present study we have demonstrated that recombinant $E$. coli expressing Mce4A protein can 
Table 2: Survival of recombinant E. coli within THP-I cells

\begin{tabular}{|c|c|c|c|}
\hline \multirow[t]{2}{*}{ Recombinant E. coli used to infect THP-I cells } & \multicolumn{3}{|c|}{ Colony forming units at different time points (\% survival) } \\
\hline & $t_{0}$ & $t_{3}$ & $t_{24}$ \\
\hline Vector only & $1426 \pm 112$ & $\begin{array}{l}376 \pm 37.74 \\
(26.3 \pm 1.8)\end{array}$ & $\begin{array}{l}6.6 \pm 1.45 \\
(0.46 \pm 0.12)\end{array}$ \\
\hline pET28a/mce4A & $3157 \pm 37.17$ & $\begin{array}{l}1493 \pm 90.07 \\
(47.2 \pm 2.7 \phi \phi)\end{array}$ & $\begin{array}{l}556 \pm 60.80 \\
\left(18.3 \pm 2.17^{\theta \theta}\right)\end{array}$ \\
\hline $\mathrm{pET} 28 \mathrm{a} /$ mce $/ A$ & $3596 \pm 106.4$ & $\begin{array}{l}2209 \pm 146.3 \\
(61.5 \pm 4.59 * *)\end{array}$ & $\begin{array}{l}770.7 \pm 59.05 \\
(21.33 \pm 1.09 \text { ***) }\end{array}$ \\
\hline
\end{tabular}

Survival was assayed as colony forming units per milliliter of lysate following lysis of the infected cells. Time points considered were as follows: $t_{0}$ I hour, $t_{3}-3$ hours and $t_{24}-24$ hours, post-infection. All values were normalised to $t_{0}$ value taken as $100 \%$. The significance was calculated using $t$ test. Recombinant $E$. coli expressing Mce4A or Mce IA survived inside THP-I cells at a significantly high CFU as compared to recombinant $E$. coli with vector alone.

$\phi \phi, p<0.005$; **, $\mathrm{p}<0.005$; when $t$ test applied to compare percentage survival of $E$. coli ( $\mathrm{pET} 28 \mathrm{a})$ with percentage survival of $E$. coli ( $\mathrm{pET} 28 \mathrm{a} / \mathrm{mce} 4 \mathrm{~A})$ and $E$. coli $(\mathrm{pET} 28 \mathrm{a} / \mathrm{mce} / \mathrm{A})$ respectively at $3 \mathrm{~h}$ of incubation.

${ }^{\theta \theta}, \mathrm{p}<0.005$; ***, $\mathrm{p}<0.0005$; $\mathrm{t}$ test applied to compare percentage survival of $E$. coli ( $\left.\mathrm{pET} 28 \mathrm{a}\right)$ with percentage survival of $E$. coli $(\mathrm{pET} 28 \mathrm{a} / \mathrm{mce} 4 \mathrm{~A})$ and E. coli $(\mathrm{pET} 28 \mathrm{a} /$ mce $/ \mathrm{A})$ respectively at $24 \mathrm{~h}$ of incubation.

enter non-phagocytic HeLa cells. Further, Mce4A protein also enables the recombinant $E$. coli to survive inside THP1 macrophages. The estimation of CFU demonstrates that the rate of intracellular survival of recombinant $E$. coli expressing $m c e 4 \mathrm{~A}$ was comparable to that of $m c e 1 \mathrm{~A}$ indicating thereby that $m c e 4 A$ may have a role similar to mce $1 \mathrm{~A}$ in invasion and survival of $M$. tuberculosis inside the host cells.

Molecular modeling studies using bioinformatics tools have shown that cell entry epitope is exposed on the surface of Mce $1 \mathrm{~A}, \mathrm{Mce} 3 \mathrm{~A}$ and Mce $4 \mathrm{~A}$ proteins but not on the Mce2A protein $[26,27]$. This suggests that the cell entry epitope of Mce1A, Mce3A and Mce4A, but not of Mce2A, may be available for interaction with mammalian cells. Subsequently a report appeared confirming the role of Mce3A and Mce3E in mammalian cell entry [12].

The recently emerging reports on the role of mce4 operon in survival of $M$. tuberculosis in host tissue indicates that Mce4 proteins may be involved in maintaining the $M$. tuberculosis in a nutrient deficient environment for long term survival [14]. It has been suggested that the proteins of the mce4 operon may operate as a major cholesterol import system of $M$. tuberculosis because strains lacking mce4 operon exhibit drastically reduced ability to take up and metabolize cholesterol in vitro and hence grow poorly when cholesterol is the primary source of carbon [14]. It was reported earlier that mutation in mce 1 operon produces growth defect in early phase of infection in mice and mutation in mce4 operon produces growth defect after 3-4 weeks post infection, indicating thereby that mce 4 operon is required in later phase of infection [28].

The localization study showed that the Mce4A protein was present in the cell wall fraction of the stationary phase cul- ture of M. tuberculosis H37Rv further strengthening our results of transmission electron microscopy and cell invasion studies.

We conclude that the results of the present study strongly support the role of Mce4A protein in invasion and the intracellular survival of $M$. tuberculosis and suggest that it may have a role in persistence of mycobacterial infection in the host tissue.

\section{Conclusion}

There are many studies reported in the literature to elucidate dormancy in M. tuberculosis and the survival mechanism of dormant tubercle bacilli is not fully understood. It is suggested that mycobacterial persistence might arise as a response to environmental stress, such as reduced oxygen concentration within host tissue. Our earlier observation of preferential expression of mce4 operon in the internal organs of rabbit and guinea pig and also during the stationary phase in culture in conjunction with the results presented here strongly support a role for Mce $4 \mathrm{~A}$ protein in the long term survival of $M$. tuberculosis inside its host.

\section{Authors' contributions}

$\mathrm{MB}$ conceived the study. $\mathrm{MB}, \mathrm{VB}$ and NKS designed the experiments, interpreted the results and worked on the manuscript. NKS carried out major experimental work. MS was associated with tissues culture studies. AC and RP participated in the designing of the experiments and the interpretation of the results. All authors read and approved the final manuscript.

\section{Acknowledgements}

Authors thank ICMR for financial support. NKS is a recipient of UGC (University grants commission), India. Senior research fellowship (No. F.2-I5/ 
2003SA-I). Authors acknowledge with thanks the electron microscopy service provided by the Department of Anatomy, AIIMS, New Delhi, India.

\section{References}

I. Corbett EL, Watt CJ, Walker N, Mahe D, Williams BG, Raviglione MC, Dye C: The growing burden of tuberculosis: global trends and interactions with the HIV epidemic. Arch Intern Med 2003 , | 63:1009-1021.

2. Frieden TR, Sterling TR, Munsiff SS, Watt CJ, Dye C: Tuberculosis. Lancet 2003, 362:887-889.

3. Flynn JL, Chan J: Immunology of tuberculosis. Ann Rev Immunol 2001, 19:93-129.

4. Bermudez LE, Goodman J: Mycobacterium tuberculosis invades and replicates within type II alveolar cells. Infect Immun 1996 , 64:1400-1406.

5. Mehta PK, Karls RK, White EH, Ades EW, Quinn FD: Entry and intracellular replication of Mycobacterium tuberculosis in cultured human microvascular endothelial cells. Microb Pathog 2006, 41:I19-124.

6. Garcia-Perez BE, Mondragon-Flores R, Luna-Herrera J: Internalization of Mycobacterium tuberculosis by macropinocytosis in non-phagocytic cells. Microb Pathog 2003, 35:49-55.

7. Shepard CC: Phagocytosis by HeLa cells and their susceptibility to infection by human tubercle bacilli. Proc Soc Exp Biol Med 1955, 90:392-396.

8. Arruda S, Bonfim G, Knights R, Huima-Byron T, Riley LW: Cloning of an $M$. tuberculosis DNA fragment associated with entry and survival inside cells. Science 1993,26 I:|454-| 457

9. Chitale S, Ehrt S, Kawamura I, Fujimura T, Shimono N, Anand N, Lu S, Gould LC, Riley L: Recombinant Mycobacterium tuberculosis protein associated with mammalian cell entry. Cell Microbiol 200I, 3:247-254.

10. Kumar A, Chandolia A, Chaudhry U, Brahmachari V, Bose M: Comparison of mammalian cell entry operons of mycobacteria: in silico analysis and expression profiling. FEMS Immunol Med Microbiol 2005, 43: 185-195.

II. Kumar M, Bose M, Brahmachari V: Analysis of expression profile of mammalian cell entry (mce) operons of Mycobacterium tuberculosis. Infect Immun 2003, 71:6083-6087.

12. Shazly SE, Ahmad S, Mustafa AS, Attiyah RA, Krajci D: Internalization by HeLa cells of latex beads coated with mammalian cell entry (Mce) proteins encoded by the mce3 operon of Mycobacterium tuberculosis. J Med Microbiol 2007, 56: I | 45- I I I I.

13. Senaratne RH, Sidders B, Sequeira P, Saunders G, Dunphy K, Marjanovic O, Reader JR, Lima P, Chan S, Kendall S, McFadden J, Riley LW: Mycobacterium tuberculosis strains disrupted in mce 3 and mce 4 operons are attenuated in mice. I of Med Microbiol 2008, 57:164-170.

14. Pandey AK, Sassetti CM: Mycobacterial persistence requires the utilization of host cholesterol. Proc Natl Acad Sci 2008 I 05:4376-4380.

15. Aguilar LD, Infante E, Bianco MV, Cataldi A, Bigi F, Pando RH: Immunogenicity and protection induced by Mycobacterium tuberculosis mce-2 and mce-3 mutants in a Balb/c mouse model of progressive pulmonary tuberculosis. Vaccine 2006, 24:2333-2342

16. Cosma CL, Sherman DR, Ramakrishnan L: The secret lives of the pathogenic mycobacteria. Annu Rev Microbiol 2003, 57:64I-76.

17. Macmicking JD, North RJ, Lacourse, Mudgett JS, Shah SK, Nathan CF: Identification of nitric oxide synthase as a protective locus against tuberculosis. Proc Natl Acad Sci 1997, 94:5243-5248.

18. Wayne LG, Sohaskey CD: Nonreplicating persistence of Mycobacterium tuberculosis. Annu Rev Microbiol 200I, 55:I39-63.

19. Via LE, Lin PL, Ray SM, Carrillo J, Allen SS, Eum SY, Taylor K, Klein E Manjunatha U, Gonzales J, Lee EG, Park SK, Raleigh JA, Cho SN, McMurray DN, Flynn JL, Barry CE III: Tuberculous granulomas are hypoxic in guinea pigs, rabbits, and nonhuman primates. Infect Immun 2008, 76:2333-2340.

20. Van Embden JD, Cave MD, Crawford JT, Dale JW, Eisenach KD, Gicquel B, Hermans P, Martin C, McAdam R, Shinnick TM: Strain identification of Mycobacterium tuberculosis by DNA fingerprinting: recommendations for a standardized methodology. J Clin Microbiol 1993, 3 I:406-409.

21. Cole ST, Brosch R, Parkhill J, Garnier T, Churcher C, Harris D, Gor don SV, Eiglmeier K, Gas S, Barry CE III, Tekaia F, Badcock K, Basham
D, Brown D, Chillingworth T, Connor R, Davies R, Devlin K, Feltwell T, Gentles S, Hamlin N, Holroyd S, Hornsby T, Jagels K, Krogh A, McLean J, Moule S, Murphy L, Oliver K, Osborne J, Quail MA, Rajandream M-A, Rogers J, Rutter S, Seeger K, Skelton J, Squares R, Squares S, Sulston JE, Taylor K, Whitehead S, Barrell BG: Deciphering the biology of Mycobacterium tuberculosis from the complete genome sequence. Nature 1998, 393:537-544.

22. Ortalo-Magne A, Dupont MA, Lemassu A, Andersen AB, Gounon $P$, Daffe $M$ : Molecular composition of the outer most capsular material of the tubercle bacillus. Microbiol 1995, |4 I:1609-1620.

23. Dahl JL, Wei J, Moulder JW, Laal S, Friedman RL: Subcellular localization of the intracellular survival-enhancing Eis protein of Mycobacterium tuberculosis. Infect Immun 200I, 69:4295-4302.

24. Harlow E, Lane D: Antibodies: A Laboratory Manual Volume 349. Cold Spring Harbor Laboratory, Cold Spring Harbor; 1988:353-355.

25. Ahmad S, Akbar PK, Wiker HG, Harboe M, Mustafa AS: Cloning, expression and immunological reactivity of two mammalian cell entry proteins encoded by the mcel operon of Mycobacterium tuberculosis. Scand J Immunol 1999, 50:510-5 I8.

26. Das AK, Mitra D, Harboe M, Nandi B, Harkness RE, Das D, Wiker HG: Predicted molecular structure of the mammalian cell entry protein McelA of Mycobacterium tuberculosis. Biochem Biophys Res Commun 2003, 302:442-447.

27. Mitra D, Saha B, Das D, Wiker HG, Das AK: Correlating sequential homology of McelA, Mce2A, Mce3A and Mce4A with their possible functions in mammalian cell entry of Mycobacterium tuberculosis performing homology modeling. Tuberculosis (Edinb) 2005, 85:337-345.

28. Sassetti CM, Rubin E]: Genetic requirements for mycobacterial survival during infection. Proc Natl Acad Sci 2003, 100:12989-12994.

Publish with Biomed Central and every scientist can read your work free of charge

"BioMed Central will be the most significant development for disseminating the results of biomedical research in our lifetime. "

Sir Paul Nurse, Cancer Research UK

Your research papers will be:

- available free of charge to the entire biomedical community

- peer reviewed and published immediately upon acceptance

- cited in PubMed and archived on PubMed Central

- yours - you keep the copyright
BioMedcentral 